\title{
Ca37 monoclonal antibody inhibits Candida albicans growth in vitro and in vivo
}

\begin{abstract}
Aitziber Antoran ${ }^{1}$, Leire Aparicioํㅜ, Idoia Buldain ${ }^{1}$, Leire Martin-Souto ${ }^{1}$, Aitana Arbizu-Delgado ${ }^{1}$, Aize Pellon$^{1}$, Aitor Rementeria ${ }^{1}$, Eleftherios Mylonakis ${ }^{2}$, Andoni Ramirez-Garcia ${ }^{1}$, Fernando L. Hernando ${ }^{1}$ ${ }^{1}$ University of the Basque Country (UPV/EHU), Fungal and Bacterial Biomics Research Group. Dept. of Immunology, Microbiology \& Parasitology, Leioa, Spain

${ }^{2}$ Division of Infectious Diseases, Rhode Island Hospital, Alpert Medical School of Brown University, 593 Eddy Street, Aldrich 708, POB 328/330, Providence, RI, USA
\end{abstract}

\section{INTRODUCTION}

Candida albicans is an opportunistic fungus, but it can cause severe infections in patients with impaired immune system. It is the first fungal agent related to health care-associated infections and the fourth causative agent of bloodstream infections. Despite the use of antifungals, mortality rates are still high (50\%). Moreover, the use of antifungals leads to toxicity and resistance to the most used drugs are emerging. Therefore, there is an urgent need to find alternative treatment options. In this sense, monoclonal antibodies (mAbs) have shown to play a protective role in animal models.

\section{OBJECTIVE}

The aim of this work was to analyze and characterize the effect of a monoclonal antibody, Ca37, directed against a metabolic protein of $C$. albicans

\section{Effect of Ca37 mAb on C. albicans growth curves}

Two strains of the fungus, NCPF 3153 and CECT 13062, were incubated in Sabouraud broth in presence of two Ca37 mAb concentrations. The growth of the reference strain, NCPF 3153, was reduced $30 \%$ in presence of $10 \mu \mathrm{g} / \mathrm{ml}$ of the $\mathrm{mAb}$. However, the growth of CECT 13062 was not affected.
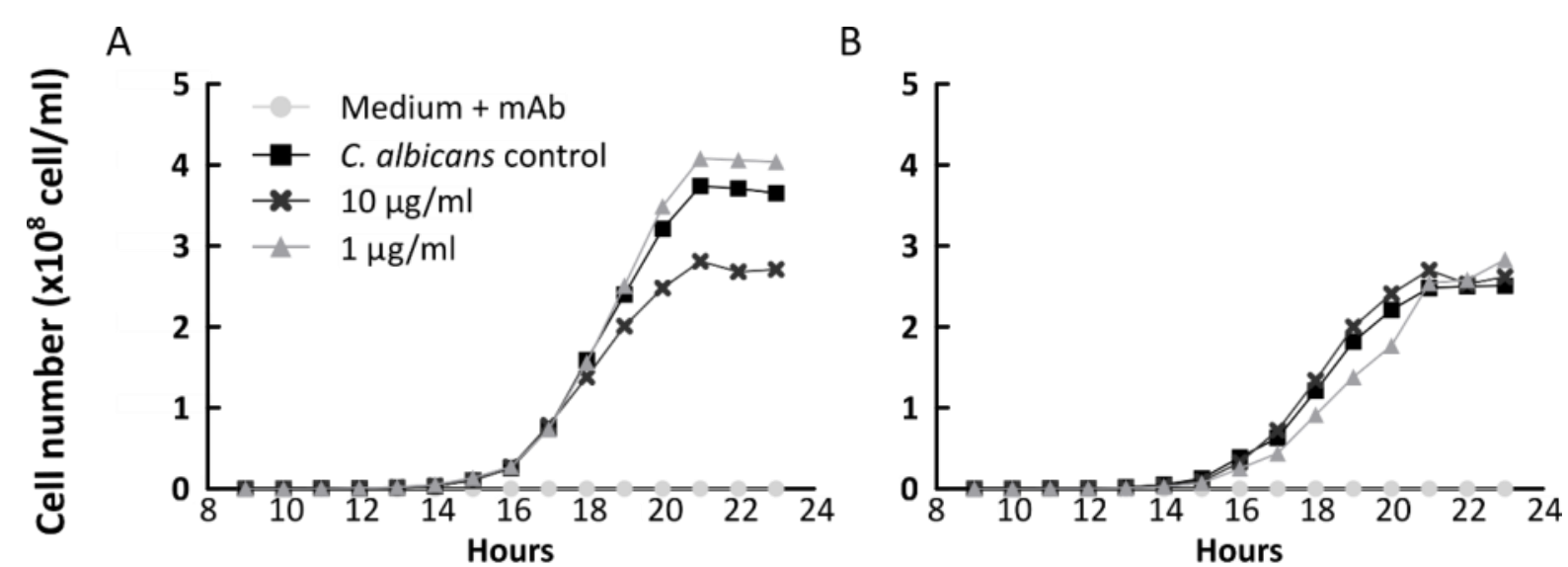

Figure 1. Growth curves of two $C$. albicans strains in presence of the mAb. (A) NCPF 3153 and (B) CECT 13062. The fungal strains were incubated with the $\mathrm{mAb}$ at $37^{\circ} \mathrm{C}$ and growth was measured for $24 \mathrm{~h}$ at $600 \mathrm{~nm}$ of absorbance. The obtained absorbance values were transform into cell densities. Mean values of the experimental replicates are shown.

\section{Reduced MIC values in combination with} antifungal drugs

\begin{tabular}{|c|c|c|c|c|c|c|c|c|c|c|c|c|c|}
\hline \multirow{2}{*}{\multicolumn{2}{|c|}{ A }} & \multicolumn{11}{|c|}{ Amphotericin B (mg/L) } & \multirow{9}{*}{$\begin{array}{l}\text { We analyze the additive } \\
\text { effect of Ca37 mAb and } \\
\text { the antifungal drugs } \\
\text { amphotericin B and } \\
\text { fluconazole following } \\
\text { the EUCAST protocol. } \\
\text { The combination of this }\end{array}$} \\
\hline & & 16 & 8 & 4 & 2 & 1 & 0.5 & \begin{tabular}{l|l}
0.25 \\
\end{tabular} & 0.125 & 0.06 & 0.03 & 0 & \\
\hline & & 0.142 & 0.102 & 0.103 & 0.106 & 0.107 & 0.106 & 0.636 & & & & & \\
\hline$\overline{\bar{\varepsilon}}$ & 20 & 0.111 & 0.109 & 0.108 & 0.106 & 0.109 & 0.108 & 0.116 & 0.544 & 0.874 & 1.124 & 0.854 & \\
\hline 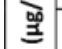 & 10 & 0.109 & 0.107 & 0.104 & 0.109 & 0.108 & 0.108 & 0.133 & 0.714 & 1.026 & 1.050 & 1.060 & \\
\hline 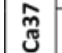 & 2 & 0.105 & 0.104 & 0.105 & 0.106 & 0.104 & 0.105 & 0.141 & 823 & 1.039 & \begin{tabular}{|l|l|l|l}
1.114 \\
\end{tabular} & 1.053 & \\
\hline & 1 & 0.103 & 0.103 & 0.102 & 0.105 & 0.106 & 0.105 & 0.140 & 0.849 & 1.028 & 0.981 & 0.993 & \\
\hline \multirow{2}{*}{\multicolumn{2}{|c|}{ B }} & \multicolumn{11}{|c|}{ Fluconazole (m//L) } & \\
\hline & & 64 & 32 & 16 & 8 & 4 & 2 & 1 & \begin{tabular}{|l|}
0.5 \\
\end{tabular} & 0.25 & 0.125 & 0 & \\
\hline & 0 & 0.136 & 0.209 & & & & & & & & & & \multirow{5}{*}{$\begin{array}{l}\text { mAb with antifungals } \\
\text { reduced MIC values to } \\
\text { half, even in the case of } \\
\text { a fluconazole-resistant } \\
\text { strain, UPV 93-153. }\end{array}$} \\
\hline \multirow{4}{*}{\multicolumn{2}{|c|}{ 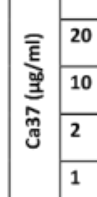 }} & 0.384 & 0.411 & 0.498 & 0.576 & 0.614 & 0.642 & 0.716 & 0.708 & 0.804 & 0.764 & 0.776 & \\
\hline & & 0.366 & 0.385 & 0.499 & 0.603 & 0.654 & 0.728 & 0.733 & 0.763 & 1.134 & 0.831 & & \\
\hline & & 0.333 & 0.411 & 0.543 & & 0.846 & 0.857 & 0.860 & 0.891 & 0.910 & 1.006 & & \\
\hline & & 0.318 & 0.314 & 0.697 & 0.959 & 0.986 & 0.986 & 0.998 & 0.992 & 0.962 & 0.982 & 1.018 & \\
\hline
\end{tabular}

Figure 3. Additive effect of $\mathrm{Ca} 37 \mathrm{mAb}$ with antifungals. Growth of $C$. albicans CECT 13062 and UPV 93-153 strains in presence of $(A)$ amphotericin $B$ and (B) fluconazole, respectively, and the $\mathrm{MAb}$. MIC values are marked in red. The grey scale indicates the percentage of inhibition.

\section{RESULTS}

\section{Inhibition of $C$. albicans growth in presence of Ca37 mAb}

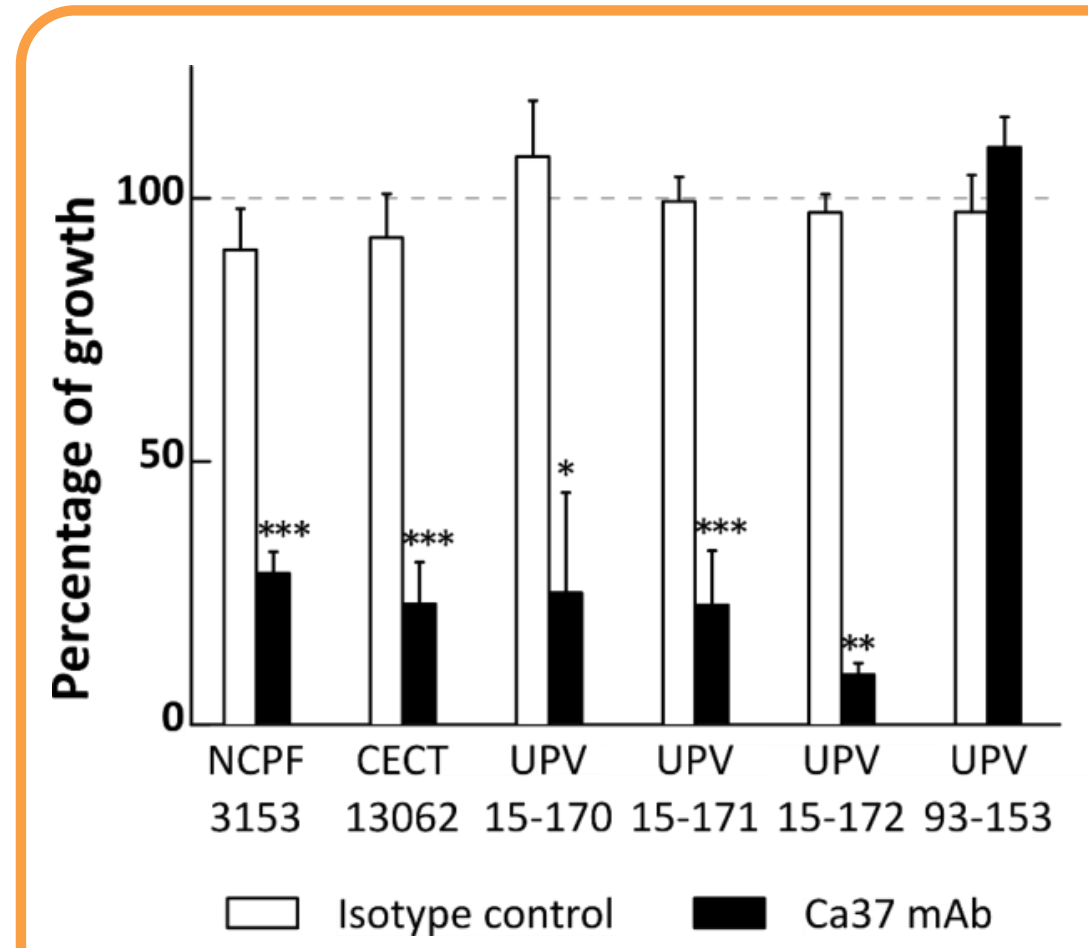

Candida albicans was incubated with different concentrations of the Ca37 mAb, and $10 \mu \mathrm{g} / \mathrm{ml}$ was the one inducing the highest inhibition of growth (data not shown).

Then, six $C$. albicans strains were incubated with this concentration and, except for the fluconazole-resistant strain UPV 93-153, the growth of all of them was reduced between $70 \%$ to $90 \%$.

Figure 2. Growth inhibition of six $C$. albicans strains in presence of $10 \mu \mathrm{g} / \mathrm{ml}$ of the mAb. Yeasts cells were incubated with the $\mathrm{mAb}$ for $18 \mathrm{~h}$ at $37^{\circ} \mathrm{C}$, and then plated for CFU counting. Statistically significant differences in comparison to the non-treated control, ${ }^{*} \mathrm{p}<0.05, * * \mathrm{p} \leq 0.01, * * * \mathrm{p} \leq 0.001$

\section{Increased survival of Galleria mellonella infected with Ca37-treated yeasts}

Candida albicans was treated with PBS, Ca37 or fluconazole for $18 \mathrm{~h}$ before been injected into the larvae, at a density of $5 \times 10^{5}$ cells/larvae.

Larvae receiving the yeast pre-treated with the mAb survived 2 days longer that the ones injected with PBS-treated fungus. The increase in survival was similar to that observed in the group receiving fluconazole treated yeasts.

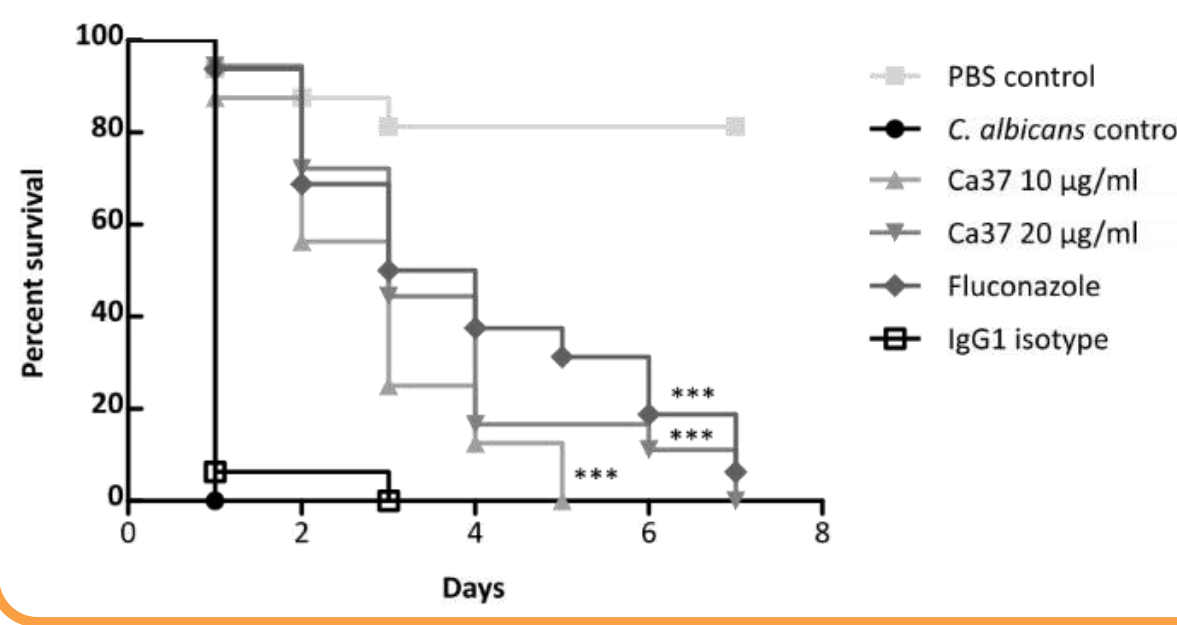

\section{CONCLUSIONS}

Ca37 is able to inhibit C. albicans growth in vitro, to reduce the dose of antifungals and to increase survival of Galleria mellonella in vivo.

The protein targeted by Ca37 mAb could be an attractive new target to fight against $C$. albicans infections.
Figure 4. Survival curves of Galleria mellonella larvae inoculated with $C$. albicans yeast pre-treated with the mAb, fluconazole or IgG1 isotype control $(n=48)$. Data from a single representative experiment. $* * * \mathrm{p} \leq 0.0001$ in comparison to non-treated $C$. albicans.

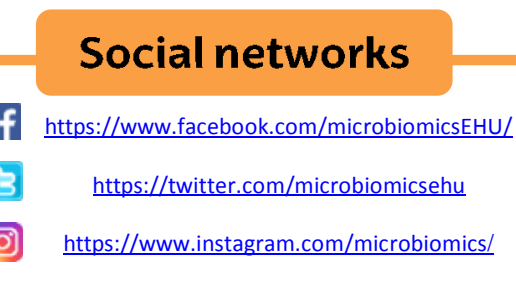

$\checkmark$ This work has been supported by grants GIU15/36 and PPG17/41 from the UPV/EHU.

$\checkmark \mathrm{AA}, \mathrm{IB}$ and LM-S were supported by predoctoral fellowship from the Basque Government, and AP by a postdoctoral fellowship from UPV/EHU. 